\title{
Super-resolution image reconstruction techniques: Trade-offs between the data-fidelity and regularization terms
}

\author{
Antigoni Panagiotopoulou, Vassilis Anastassopoulos \\ Electronics Laboratory, Physics Department, University of Patras, Rio 26500, Greece \\ Phone: +302610996147 Fax: +302610997456 E-mail: vassilis@ physics.upatras.gr
}

\begin{abstract}
Stochastic regularized methods are quite advantageous in Super-Resolution (SR) image reconstruction problems. In the particular techniques the SR problem is formulated by means of two terms, the data-fidelity term and the regularization term. The present work examines the effect of each one of these terms on the SR reconstruction result with respect to the presence or absence of noise in the Low-Resolution (LR) frames. Experimentation is carried out with the widely employed $L_{2}, L_{1}$, Huber and Lorentzian estimators for the datafidelity term. The Tikhonov and Bilateral (B) Total Variation (TV) techniques are employed for the regularization term. The extracted conclusions can, in practice, help to select an effective SR method for a given sequence of LR frames. Thus, in case that the potential methods present common data-fidelity or regularization term, and frames are noiseless, the method which employs the most robust regularization or data-fidelity term should be used. Otherwise, experimental conclusions regarding performance ranking vary with the presence of noise in frames, the noise model as well as the difference in robustness of efficiency between the rival terms. Estimators employed for the data-fidelity term or regularizations stand for the rival terms.
\end{abstract}

Index terms: super-resolution, data-fidelity, regularization, noisy frames, method selection

\section{Introduction}

Super-resolution is the term generally applied to the problem of transcending the limitations of optical imaging systems by employing image processing algorithms. Basically, in SR image reconstruction a sequence of noisy blurred LR images are fused to produce a higherresolution image, which exhibits more high-frequency content and less noise and blur effects than any of the employed LR images. Actually, the obtained High-Resolution (HR) image contains pieces of information from all the LR images. Therefore, data fusion takes place. Early works on SR reconstruction have shown that the recovery of the HR fused image is enabled by the aliasing effects that exist in the LR images, as long as there is a relative subpixel shift between the particular images [1]. The resolution enhancement results of SR image reconstruction methods [2-4] are more powerful than those of interpolation techniques [5-6]. 
Several approaches to the SR image reconstruction problem have been developed [4, 7]. The stochastic regularized SR techniques are quite advantageous. In these techniques the formulation of the SR problem takes place by means of two terms, the data-fidelity term and the regularization term. In literature the $L_{2}, L_{1}$, Huber and Lorentzian estimators are commonly employed for the data-fidelity term. Additionally, the Tikhonov and bilateral TV regularization techniques are often utilized for the regularization term [3, 8-14]. Often, for a given sequence of LR frames, the most effective SR method among several potential ones has to be chosen before proceeding to the SR reconstruction task. If there is common data-fidelity or regularization term in the methods, and the LR frames are noiseless, the method which presents the most robust regularization or data-fidelity term should be employed. Nevertheless, if the frames are corrupted by noise and/or there are different data-fidelity terms as well as different regularization terms, selecting the most effective SR method is ambiguous. In literature a variety of SR reconstruction methods have been presented. Nevertheless, there has not yet been presented any work dealing with the selection of an effective SR method, among several potential ones, for a given LR sequence of frames. The present work treats this specific issue.

In the present work trade-offs between the data-fidelity and regularization terms are considered. Actually, these terms effect on the SR reconstruction result is worked out. The $L_{2}, L_{1}$, Huber and Lorentzian estimators are employed for measuring the difference between the projected estimate of the HR image and each LR frame. Regularization takes the form of the Tikhonov and Bilateral TV (BTV) priors. Eight different SR methods are formulated. Experimentation is carried out with noiseless frames as well as with frames corrupted by noise of various models. The experimental results are evaluated and conclusions are reached via grouping the SR methods. The methods are grouped per common data-fidelity term and per common regularization term. Grouping of the methods per pairs takes place as well. In the specific grouping, each pair of methods presents rival terms that exhibit different robustness of efficiency. Estimators employed for the similarity cost or regularizations stand for the rival terms. The conclusions reached can, in practice, help to select an effective SR image reconstruction method for a given sequence of LR frames. Therefore, in case that the potential SR methods present common data-fidelity term the method employing the most robust regularization should be chosen. If the methods display common regularization term and frames are noiseless, the ranking in methods performance is in accordance with the ranking in robustness of the estimators employed for assuring fidelity to the data. Nevertheless, in case that the poten- 
tial methods exhibit different data-fidelity terms as well as different regularization terms, experimental conclusions regarding performance ranking vary. In the particular case the ranking in methods performance is affected by the absence or presence of noise in frames, the noise model and the difference in robustness of efficiency between the rival terms.

In Sections 2 and 3 the data-fidelity and regularization terms that formulate the SR image reconstruction problem are discussed. The experimental procedure is presented in Section 4. The experimental results are provided in Section 5. Conclusions are drawn in Section 6.

\section{Data-fidelity term}

Super-resolution image reconstruction algorithms attempt to extract the HR image corrupted by the limitations of the optical imaging system. Before proceeding to solving the specific inverse problem, a forward model has to be formed. The most commonly employed forward model is linear and presents the following form

$$
Y(t)=M(t) X(t)+V(t) .
$$

The operator $Y$ stands for the measured LR images, while $X$ is the uknown HR image. The operator $M$ represents the imaging system and $V$ is the random noise inherent to any image acquisition system. The symbol $t$ denotes the time of image acquisition [15]. By the employment of a forward model, a cost function has to be defined for the estimation of $X$. The particular cost function, named $\rho$-function or error norm, assures a certain fidelity or closeness of the final solution to the measured data. Actually, an error norm is used to measure the difference between the projected estimate of the HR image and each LR frame. The robustness of efficiency of the employed estimator [16-17] plays a vital role in the SR reconstruction results. Thus, the choice of the particular $\rho$-function is critical. To analyze the behavior of a given $\rho$-function its influence function $\psi$, which is proportional to the derivative of $\rho$, is considered. The influence function characterizes the bias that a particular measurement has on the solution [16]. The $L_{2}, L_{1}$, Huber and Lorentzian estimators are commonly employed for the data-fidelity term in literature [3, 8-14]. For evaluating the performance of these estimators in the SR reconstruction task, their performance in rejecting outliers is of interest.

With regard to the $L_{2}$ estimator, its $\rho$-function has a linear $\psi$-function. A least-squares estimate is very sensitive to outliers because the influence function increases linearly and without bound. To increase robustness and reject outliers, the $\rho$-function must be more forgiving about outliers, that is it should increase less rapidly than $x^{2}$. Such a $\rho$-function is 
that of the $L_{1}$-norm estimator. The $L_{1}$-norm converges to median estimation. As far as the Huber norm is concerned, it is an error norm from the robust statistics literature. The Huber estimator is essentially the least-squares estimator, but uses the $L_{1}$-norm for points that are considered outliers with respect to a certain threshold which is called Huber parameter. The linear growth for large $x$ makes approximation less sensitive to outliers. The Huber error norm and its influence function are given by

$$
\begin{aligned}
& \rho_{H b}(x)=\left\{\begin{array}{c}
x^{2}, \quad|x| \prec \text { hubpar } \\
\text { 2hubpar }|x|-\text { hubpar }^{2}, \quad \text { otherwise }
\end{array}\right. \\
& \psi_{H b}(x)=\left\{\begin{array}{c}
2 x, \quad|x| \prec \text { hubpar } \\
\text { 2hubparsign }(x), \text { otherwise }
\end{array}\right.
\end{aligned}
$$

where hubpar is the Huber parameter. The influence curve of the Huber estimator is constant for all observations beyond a certain point. An $M$-estimator can be made more resistant by having the $\psi$-function, and hence the influence curve, return to 0 . Such an estimator is called a redescending estimator. Outliers have diminishing effects on a redescending estimator. The Lorentzian error norm is such an estimator. The Lorentzian $\rho$ - and $\psi$-functions are given below, where lorpar is the scale parameter.

$$
\begin{gathered}
\rho_{L r}(x)=\log \left[1+\frac{1}{2}\left(\frac{x}{\text { lorpar }}\right)^{2}\right] \\
\psi_{L r}(x)=\frac{2 x}{2 \text { lorpar }^{2}+x^{2}}
\end{gathered}
$$

\section{Regularization term}

\subsection{The concept of regularization in Mathematics}

In general terms regularization is the approximation of an ill-posed problem by a family of neighbouring well-posed problems. Let it is required to approximate the best-approximate solution $x^{\dagger}=T^{\dagger} y$ of

$$
T x=y
$$

for a specific right-hand side $y$ in the situation that the exact data $y$ are not known precisely, but that only an approximation $y^{\delta}$ with 


$$
\left\|y^{\delta}-y\right\| \leq \delta
$$

is available. $y^{\delta}$ is called noisy data, whilst $\delta$ is called noise level. In the ill-posed case $T^{\dagger} y^{\delta}$ is certainly not a good approximation of $T^{\dagger} y$ due to the unboundness of $T^{\dagger}$ even if it exists. $T^{\dagger}$ means the transpose of the complex conjugate of $T$. It is required to find an approximation, say $x_{a}^{\delta}$, of $x^{\dagger}$. The particular approximation should depend continuously on the noisy data $y^{\delta}$, so that it can be computed in a stable way. Additionally, it should have the property that, as the noise level $\delta$ decreases to zero and the regularization parameter $a$ is chosen appropriately, then $x_{a}^{\delta}$ tends to $x^{\dagger}[18-19]$.

In general the construction of $x_{a}^{\delta}$ involves the operator $T$ [18]. Thus, it makes more sense to look not only at the equation (6) for one specific right-hand side $y$, but to consider (6) as a collection of equations for every $y \in R(T)$ or $y \in D\left(T^{\dagger}\right)$. The latter notations mean that the equations $T x=y$ or $x^{\dagger}=T^{\dagger} y$ hold respectively. Then, we are not only talking about regularizing a specific equation, but this collection of equations or about regularizing the solution operator $T^{\dagger}$. Then, intuitively a regularization of $T^{\dagger}$ should be the replacement of the unbounded operator $T^{\dagger}$ by a parameter-dependent family $\left\{R_{a}\right\}$ of continuous operators. As approximation of $x^{\dagger}$ we then take $x_{a}^{\delta}:=R_{a} y^{\delta}$, which can be computed in a stable way. The regularization parameter $a$ has to be somehow linked with $\delta$ and/or $y^{\delta}$, maybe with other information about $T$ or $y$ as well. Such parameter choice rules should be linked with either $y^{\delta}$ or some a-priori information about the exact data $y$. Since $y$ is not known, this dependence can only be on some qualitative a-priori knowledge about $y$, like smoothness properties. Therefore, regularization operators are defined for the whole collection of equations

$$
T x=y, \quad y \in D\left(T^{\dagger}\right)
$$

or for the operator $T$, but parameter choice rules are defined for a specific equation out of this collection. Both together form a regularization method for solving one specific equation.

\subsection{Regularization in super-resolution image reconstruction}

Super-resolution image reconstruction is an ill-posed problem. Thus, considering regularization in the SR method as a means for picking a stable solution is very useful, if not necessary. Additionally, regularization can serve for removing artifacts from the final answer 
and improving the rate of convergence. A regularization term compensates the missing measurement information with some general prior information about the desirable HR solution. Traditionally, regularization has been described from both the algebraic and statistical perspectives, and takes the form of constraints on the space of possible solutions. The imposition of these constraints is accomplished via Lagrangian type penalty terms as in

$$
J(X)=\|Y-M X\|_{2}^{2}+\lambda \rho(X) .
$$

The function $\rho(X)$ poses a penalty on the unknown $X$ in order to direct it to a better formed solution. The coefficient $\lambda$, called regularization parameter, determines the enforcement strength of the particular penalty. The choice of $\lambda$ can be done either manually, via visual inspection, or automatically employing methods like generalized cross-validation and $L$ - curve methods [20-22] or Bayesian estimation method [23].

The Tikhonov regularization belongs to the early efforts regarding regularization and is widely referenced. It has the form

$$
\rho_{T i k h}(X)=\|\Gamma X\|_{2}^{2}
$$

where $\Gamma$ is an operator capturing some aspect of the image, like its general smoothness. It is usually a highpass operator such as derivative, Laplacian or even identity matrix. The intuition behind this regularization is to limit the total energy of the image or force spatial smoothness. Penalization of energy in the higher frequencies of the solution takes place. The noisy and edge pixels both contain high-frequency energy. The Tikhonov regularization process removes them and thus, the resulting image does not contain sharp edges.

From a statistical perspective employing the MAP estimator regularization is incorporated as a-priori knowledge about the solution. Unlike the traditional Tikhonov penalty terms, robust regularization techniques present the capability of performing adaptive smoothing based on the local structure of the image. The bilateral total variation regularization is a robust regularization technique. The BTV regularizer is given by

$$
\rho_{B T V}(X)=\sum_{l=-P}^{P} \sum_{m=0}^{P} \alpha^{|m|+|l|}\left\|X-S_{x}^{l} S_{y}^{m} X\right\|_{1} \text {. }
$$

The operators $S_{x}^{l}$ and $S_{y}^{m}$ shift the image $X$ by $l$ and $m$ pixels horizontally and vertically respectively presenting several scales of derivatives. The weight $\alpha$ is a scalar taking values between 0 and 1 and gives a spatially decaying effect to the regularization terms summation. The parameter $P$ determines the size of the regularization kernel. The BTV regularization is 
based on the spirit of the TV restoration model [24] and the bilateral filter [25]. The TV anisotropic diffusion model is one of the most successful tools for image restoration and edge enhancement. The bilateral filter removes noise from images, keeping sharp edges. By applying the bilateral filter several times, the signal is smoothed and gets to a steady state.

The Tikhonov and bilateral TV regularization techniques are often employed for the SR image reconstruction task. Unlike bilateral TV regularization, increasing the number of iterations in Tikhonov regularization results in more undesired smoothing. Thus, the Tikhonov regularization functional exhibits the tendency to remove point like details from the image. The BTV prior not only produces sharp edges but retains point like details as well.

\section{Experimental procedure}

In this work experimentation is carried out to assess the importance of each one of the datafidelity and regularization terms in affecting the SR image reconstruction result, with respect to the presence or absence of noise in the LR frames. Various noise models are considered. An HR image is created from a sequence of subpixel shifted, aliased LR frames. Resolution is increased by a factor of 4 . The employed error norms and priors are given in Table I. The $L_{2}$, $L_{1}$, Huber and Lorentzian estimators serve for estimating the difference between the projected estimate of the HR image and each LR frame. Regularization takes the form of the Tikhonov and bilateral TV priors. The study carried out in the present work requires the employment of regularizers which present different robustness of efficiency. The two regularizers employed, Tikhonov and BTV, do exhibit different robustness of efficiency. No additional regularizer needs to be considered throughout experimentation. Eight different SR methods are formed by combining the aforementioned estimators and regularizers. The particular methods are listed in Table I. The gradient-based steepest descent method performs the minimization task of solving the SR image reconstruction problem [14]. Evaluation of the experimental results takes place and conclusions are reached via grouping the tested SR methods. Thus, the methods are grouped per common data-fidelity term as well as per common regularization term. Grouping of the methods per pairs also takes place. In the particular type of grouping, six pairs of methods are formed. Each pair of methods presents rival terms (data-fidelity or regularization terms) which exhibit different robustness of efficiency.

Several simulated experiments take place. At each experiment SR image reconstruction is applied to a synthesized LR sequence which consists of 16 frames. A LR image sequence is created from the original HR image through the following procedure. At the beginning, in or- 
der to simulate the effect of camera PSF, the HR image is convolved with a symmetric Gaussian low-pass filter of size $4 \times 4$ and standard deviation equal to 1 . The image that results is then downsampled by the factor of 4 in each of the horizontal and vertical directions through pixel extraction. The prementioned procedure, preceded by subpixel shifting the HR image in the vertical and horizontal directions employing various motion vectors, is followed to produce 15 LR images from the original HR image. Experiments are conducted with noiseless frames as well as with frames corrupted by salt\&pepper, Gaussian, speckle and Poisson noise, for two different scenes. Fig. 1 and 2 depict data regarding the Stanford and Lena scenes, respectively. With regard to noisy experimentation, salt\&pepper noise of density equal to 0.05 , Gaussian white noise of mean 0 and variance 0.01 as well as speckle noise with $V=0.04$ are employed. The experiments are implemented in Matlab.

All the conducted experiments are synthesized experiments. However, outliers are present in the experiments employing noiseless frames as well as in those employing noisy frames. Outliers are defined as data points with different distributional characteristics than those mandated by the assumed image acquisition model [8]. In the noiseless experiments outliers result from the original HR image shifting task. The motion vectors associated with each LR frame are known. Nevertheless, an interpolation-based shifting procedure lacking accuracy is followed, which essentially introduces a bias. As far as the noisy experiments are concerned, the noise introduced in the LR frames is ignored in the image acquisition model formulated for performing the SR reconstruction. Therefore, outliers are also present in these experiments.

In the present work the Lorentzian, Huber, $L_{1}$ and $L_{2}$ - error norms, in combination with the BTV and Tikhonov regularizations, are directly compared in performing the task of SR image reconstruction, thus in rejecting outliers. The direct comparison of the Lorentzian and Huber error norms, through their influence functions, requires to dilate and scale the functions to make them as similar as possible. Thereafter, first the values of the scale parameters hubpar and lorpar are chosen so that to dilate each of the two influence functions in order to begin rejecting outliers at the same value par [17]. It is set hubpar=par and lorpar $=\frac{\text { par }}{\sqrt{2}}$. Second, the two influence functions are scaled so that to return values in the same range. As far as the $L_{1}$ and $L_{2}$-norms are concerned, there is no scale parameter involved. Regarding scaling, the particular influence functions are scaled as the rest ones.

The "robust scale" par of the image is estimated as described in [14]. The values of the parameter par estimated for the Lena scene are given in Table II. Table III presents the val- 
ues of the gradient step size, denoted $\beta$, and the values of the regularization term parameters $a, \lambda$ employed by the tested methods for the Lena scene. Additionally, $\Gamma=\frac{1}{8}\left[\begin{array}{ccc}1 & 1 & 1 \\ 1 & -8 & 1 \\ 1 & 1 & 1\end{array}\right]$ is used for Tikhonov regularization whilst the size $P$ of the BTV regularization kernel is set equal to 2. With regard to the first approximation $X_{0}$ of the desired HR image, in the noiseless frames case a bilinearly interpolated LR frame serves for the initialization $X_{0}$. In the noisy frames case, a bilinearly interpolated "clean" LR frame is utilized. The particular "clean" frame is created by applying a median estimation procedure at the noisy frames.

At the presented experiments the parameters to deal with are $\beta, p a r, a$ and $\lambda$. Each method requires a different value for the parameter $\beta$. Regarding the parameter par, it has a common value for the Lorentzian and Huber estimator methods. Also, the regularization term parameters $a, \lambda$ have common value for all methods. In fact, experimentation shows that the direct comparison strictly demands common values for the parameters $a, \lambda$. Moreover, the experimentation carried out shows that it is difficult to formulate an automatic/semi-automatic parameter estimation procedure. In fact, the value of the parameter par has to be estimated by means of standard techniques which estimate the outliers rejection threshold. Regarding the parameters $\beta, a$ and $\lambda$, their values have to be specified manually through visual monitoring [14]. Additionally, apart from visual inspection, the two numerical measures Xydeas and Petrovich [26] as well as MSE are employed to assess the methods SR performance. The Xydeas and Petrovich measure is an objective test of edge information between two images and takes values in the range $[0,1]$. Its value is 0 when the images under comparison do not share any edge information, whereas its value is 1 when there is no loss of edge information.

Table I: The employed error norms and priors along with the formed SR methods.

\begin{tabular}{|l|l|l|}
\hline Data-fidelity term & Regularization term & \multicolumn{1}{|c|}{ SR methods } \\
& & $L_{2}+$ Tikhonov \\
$L_{2}-$ norm & & $L_{2}+\mathrm{BTV}$ \\
$L_{1}-$ norm & Tikhonov prior & $L_{1}+$ Tikhonov \\
Huber norm & BTV prior & $L_{1}+\mathrm{BTV}$ \\
Lorentzian norm & & Huber+Tikhonov \\
& & Huber+BTV \\
& & Lorentzian+Tikhonov \\
& & Lorentzian+BTV \\
\hline
\end{tabular}


Table II: The parameter par utilized by the Huber and Lorentzian methods for the Lena scene.

\begin{tabular}{|l|l|l|l|l|l|}
\hline Scene/Data & Noiseless & Salt\&pepper & Gaussian & Speckle & Poisson \\
\hline Lena & 0.0119 & 0.0237 & 0.1216 & 0.1260 & 0.0549 \\
\hline
\end{tabular}

Table III: The parameters $\beta / a / \lambda$ employed by the tested methods for the Lena scene.

\begin{tabular}{|l|c|c|c|c|c|}
\hline \multicolumn{1}{|c|}{ SR method/Data } & Noiseless & Salt\&pepper & Gaussian & Speckle & Poisson \\
\hline$L_{2}+\mathrm{BTV}$ & $0.1 / 0.2 / 0.009$ & $0.1 / 0.2 / 0.009$ & $0.1 / 0.8 / 0.009$ & $0.1 / 0.8 / 0.009$ & $0.1 / 0.2 / 0.009$ \\
\hline$L_{2}+$ Tikhonov & $0.1 /-/ 0.009$ & $0.1 /-/ 0.009$ & $0.1 /-/ 0.009$ & $0.1 /-/ 0.009$ & $0.1 /-/ 0.009$ \\
\hline$L_{1}+\mathrm{BTV}$ & $0.01 / 0.2 / 0.009$ & $0.01 / 0.2 / 0.009$ & $0.01 / 0.8 / 0.009$ & $0.01 / 0.8 / 0.009$ & $0.01 / 0.2 / 0.009$ \\
\hline$L_{1}+$ Tikhonov & $0.01 /-/ 0.009$ & $0.01 /-/ 0.009$ & $0.01 /-/ 0.009$ & $0.01 /-/ 0.009$ & $0.01 /-/ 0.009$ \\
\hline Huber+BTV & $0.03 / 0.2 / 0.009$ & $0.03 / 0.2 / 0.009$ & $0.03 / 0.8 / 0.009$ & $0.03 / 0.8 / 0.009$ & $0.03 / 0.2 / 0.009$ \\
\hline Huber+Tikhonov & $0.03 /-/ 0.009$ & $0.03 /-/ 0.009$ & $0.03 /-/ 0.009$ & $0.03 /-/ 0.009$ & $0.03 /-/ 0.009$ \\
\hline Lorentzian+BTV & $0.02 / 0.2 / 0.009$ & $0.02 / 0.2 / 0.009$ & $0.02 / 0.8 / 0.009$ & $0.02 / 0.8 / 0.009$ & $0.02 / 0.2 / 0.009$ \\
\hline Lorentzian+Tikhonov & $0.02 /-/ 0.009$ & $0.02 /-/ 0.009$ & $0.02 /-/ 0.009$ & $0.02 /-/ 0.009$ & $0.02 /-/ 0.009$ \\
\hline
\end{tabular}

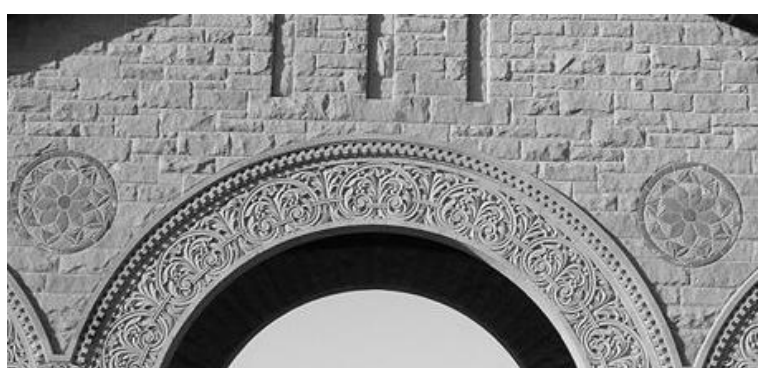

a

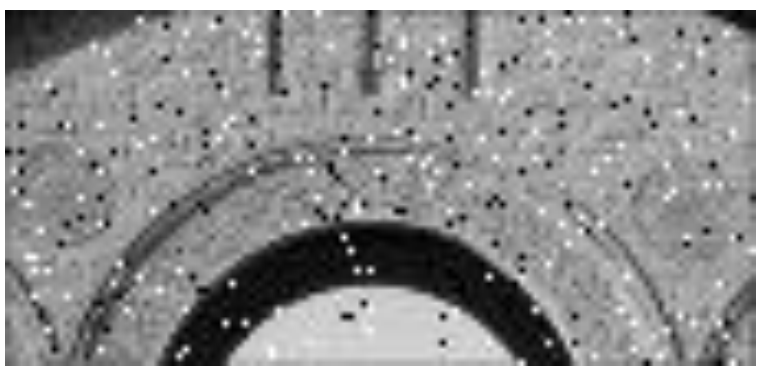

c

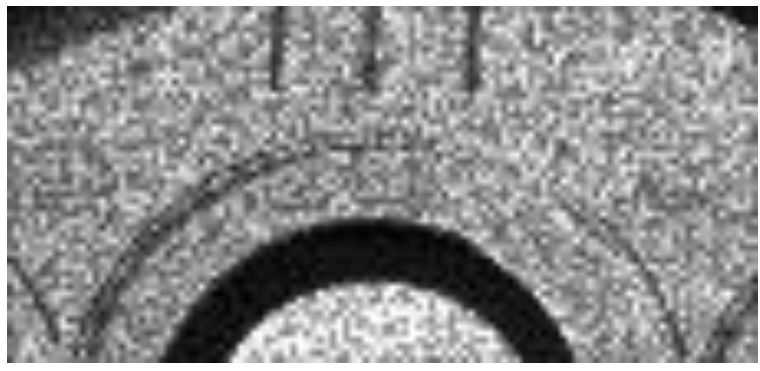

e

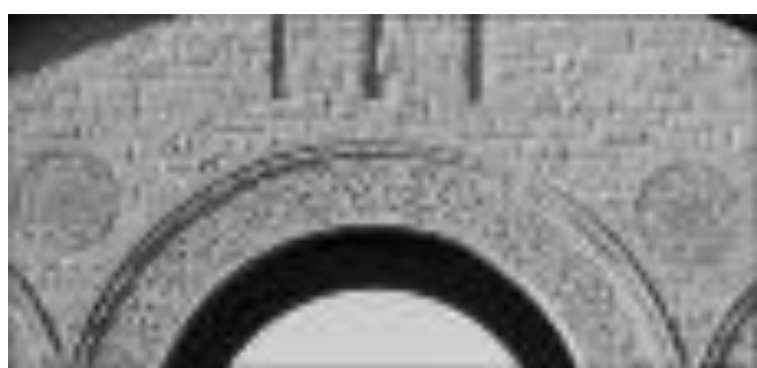

b

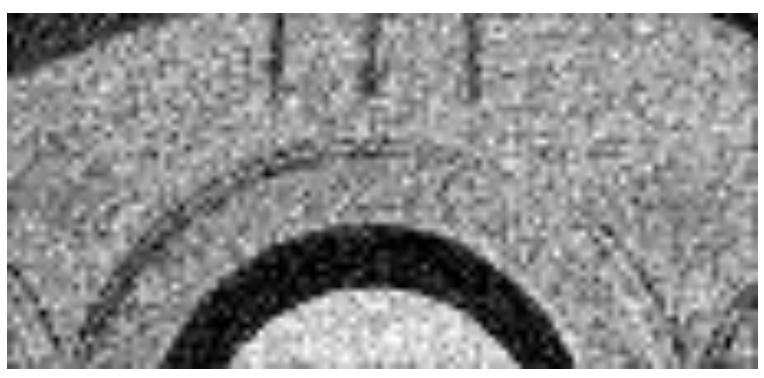

d

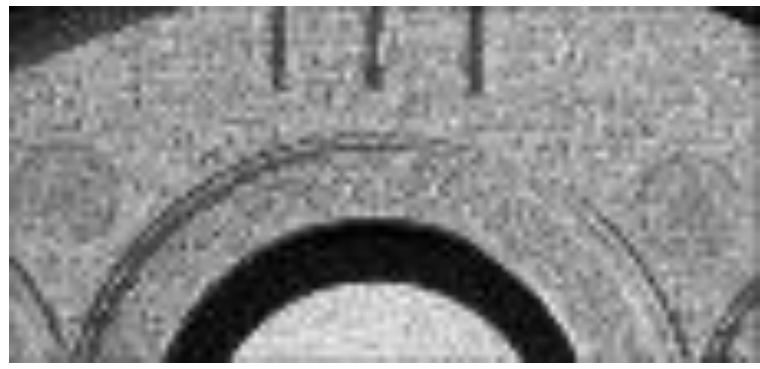

$f$

Fig. 1. Data regarding the Stanford scene. a) The original HR image. b) A frame of the LR sequence. c) Salt\&pepper noisy frame. d) Gaussian noisy frame. e) Speckle noisy frame. f) Poisson noisy frame. 


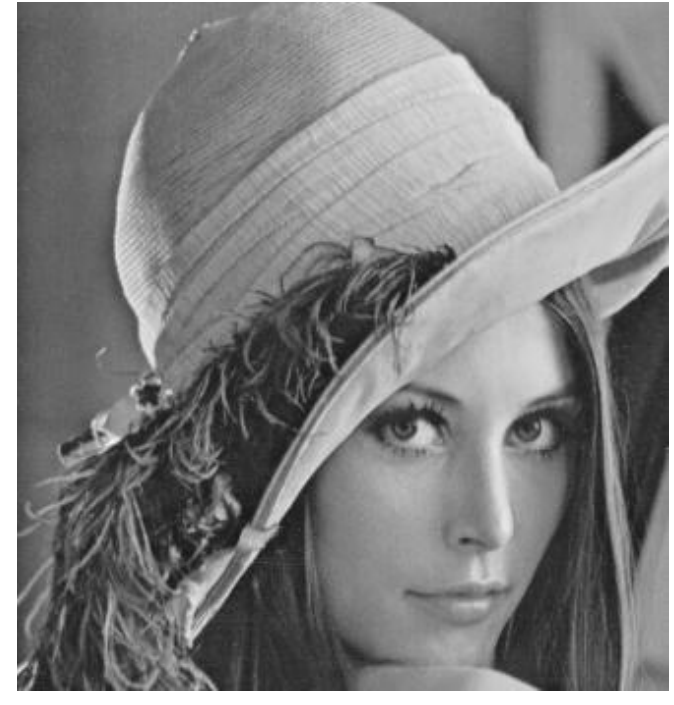

a

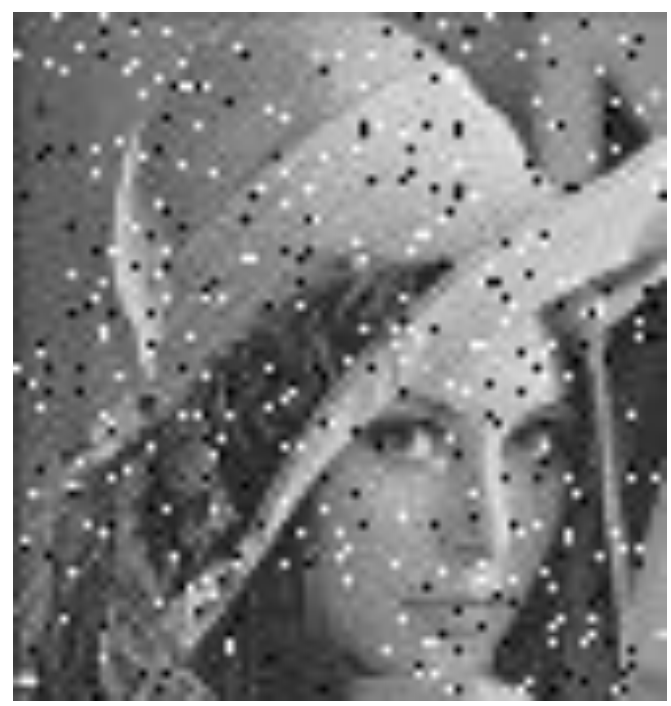

c

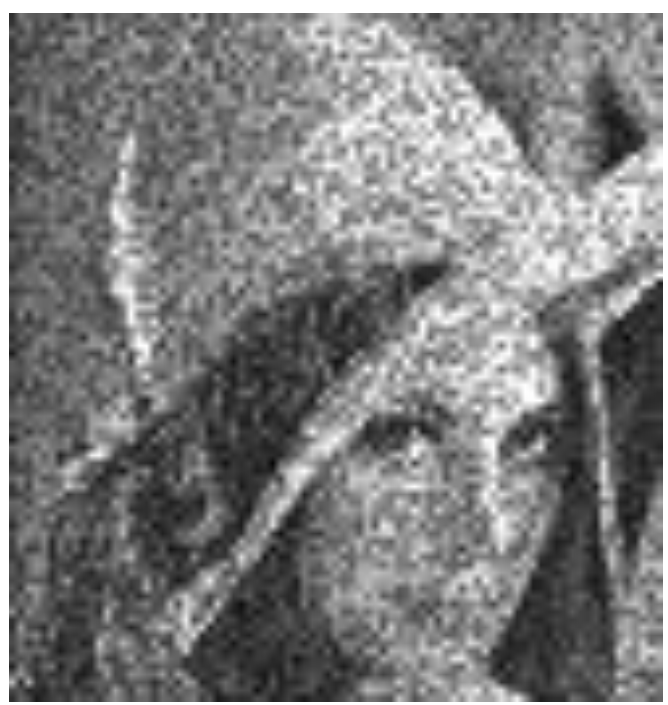

e

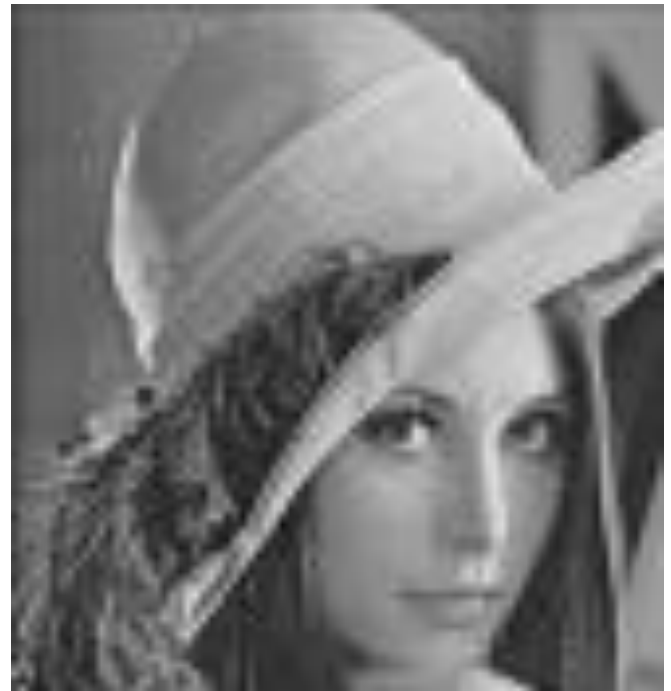

$\mathrm{b}$

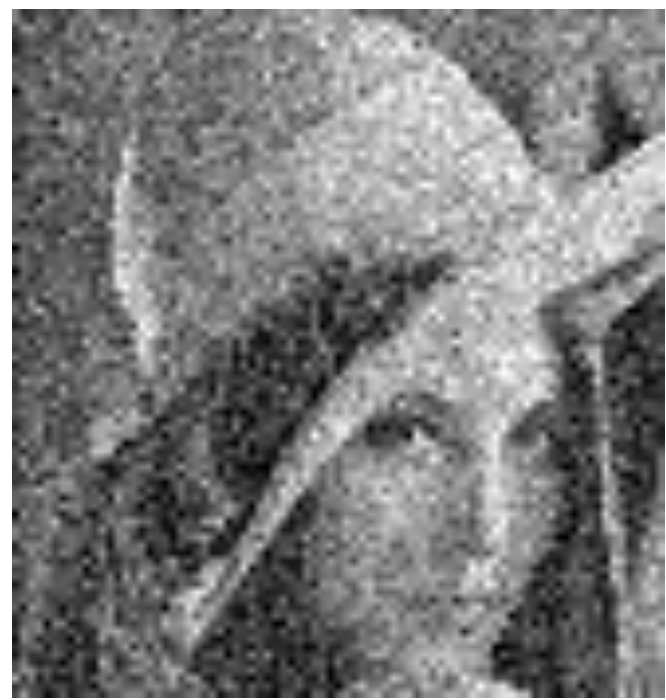

$\mathrm{d}$

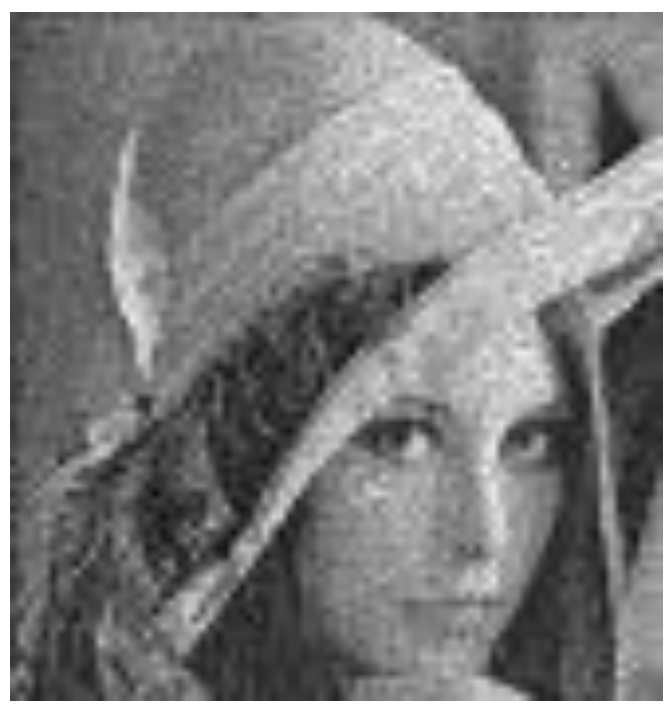

f

Fig. 2. Data regarding the Lena scene. a) The original HR image. b) A frame of the LR sequence. c) Salt\&pepper noisy frame. d) Gaussian noisy frame. e) Speckle noisy frame. f) Poisson noisy frame. 


\section{Experimental results \\ 5.1. Numerical results}

Fig. 3 and 4 depict SR reconstructed Lena images obtained for noiseless frames and frames corrupted by salt\&pepper noise, correspondingly. Tables IV, V, VI, VII and VIII present the numerical results obtained for the tested SR methods in all cases of experimentation. The values of the Xydeas and Petrovich [26], and MSE measures are presented. Evaluation of the experimental results can take place via grouping the tested methods, along with their results.

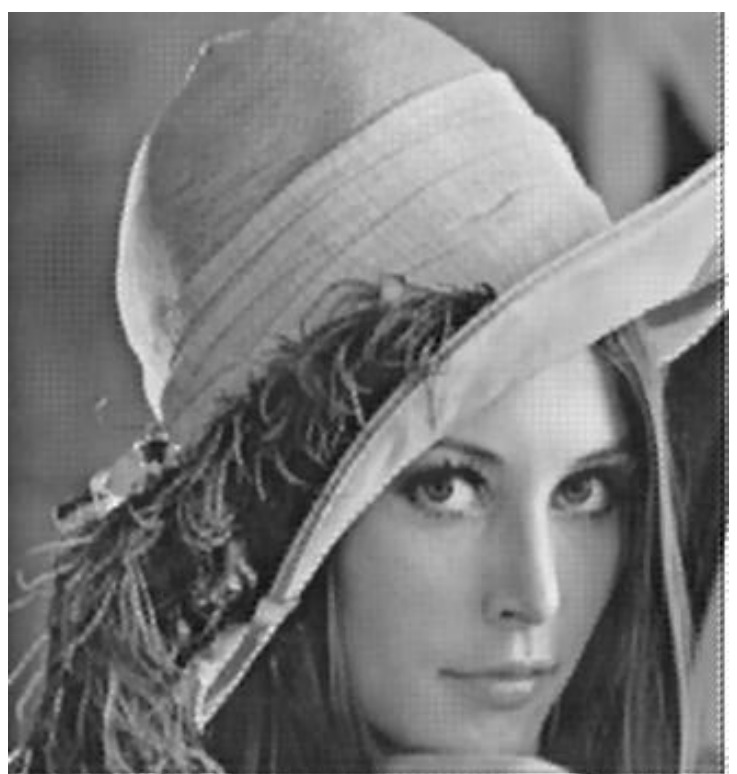

$a$

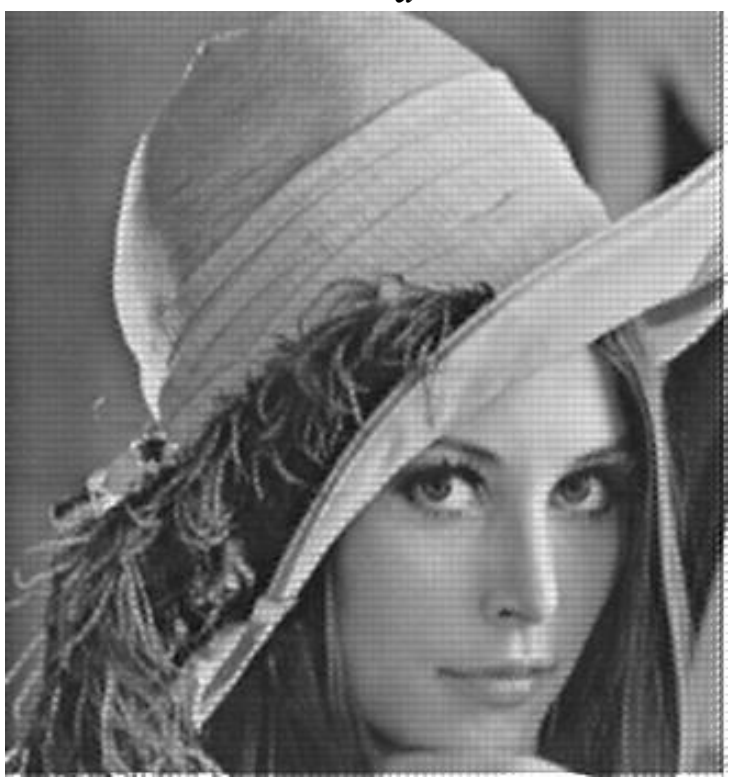

$c$

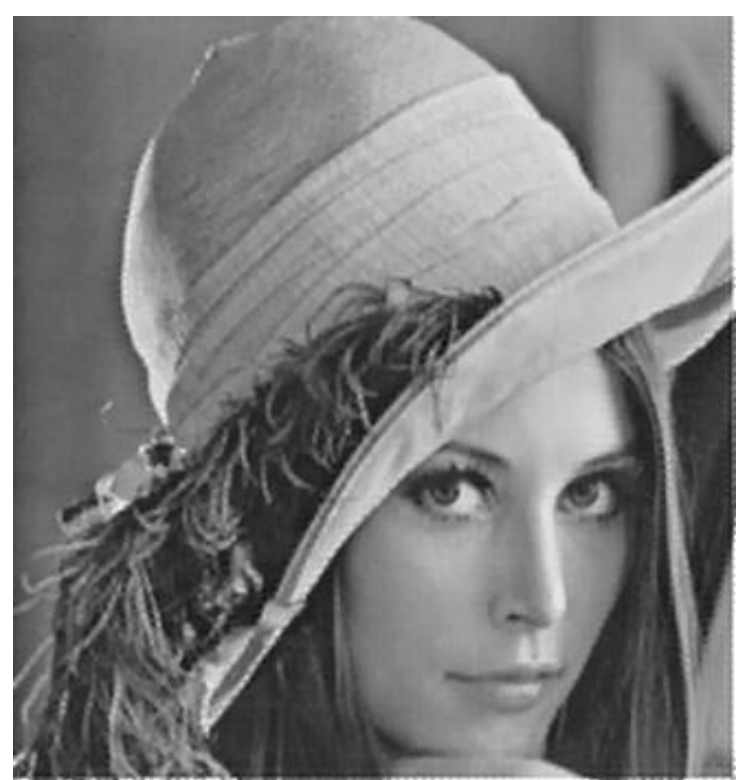

$b$

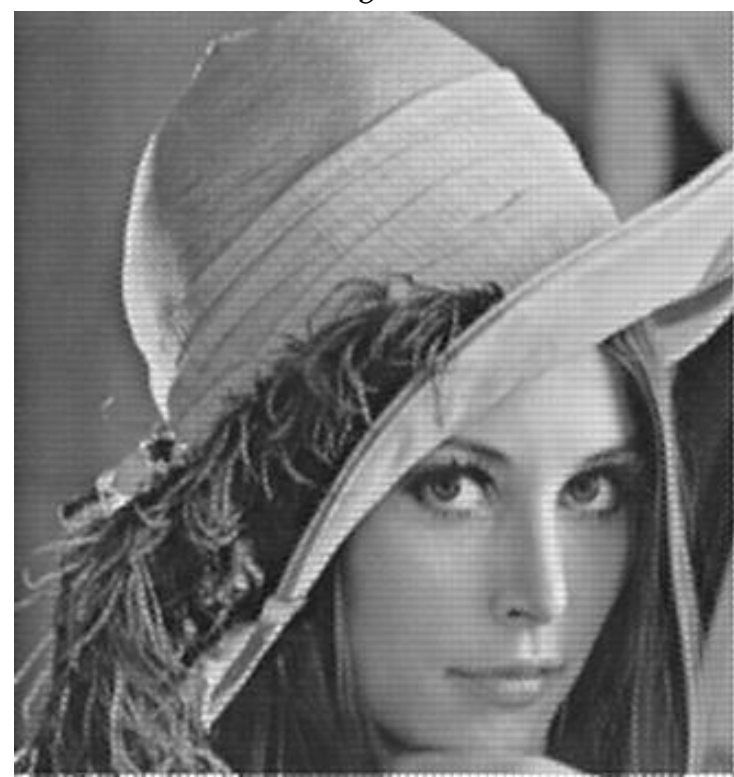

$d$

Fig. 3. SR reconstructed Lena images in case of noiseless sequence of frames. a) $L_{2}+B T V$ technique. b) Lorentzian+BTV technique. c) $L_{2}+$ Tikhonov technique. d) Lorentzian+Tikhonov technique. 


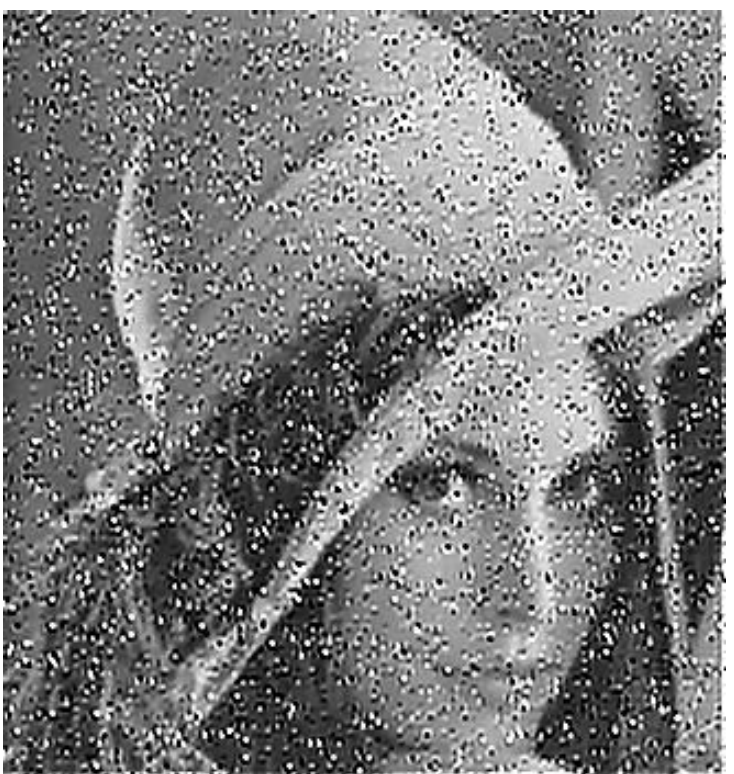

$a$

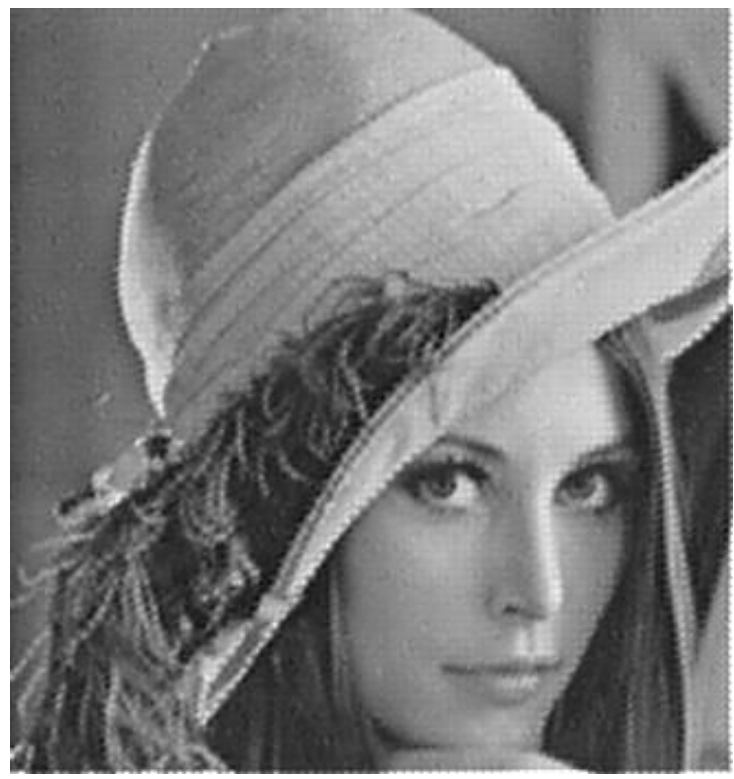

$c$

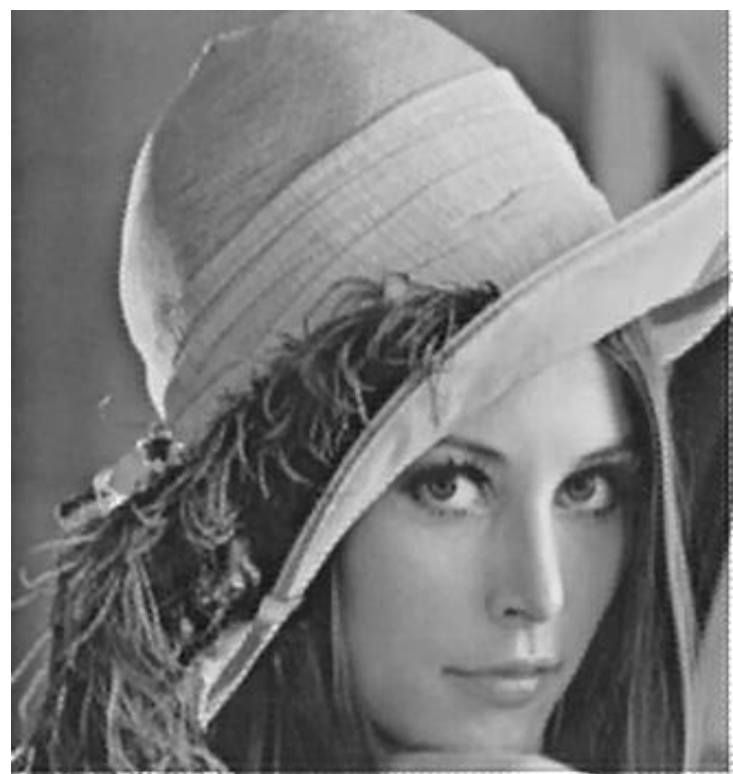

$b$

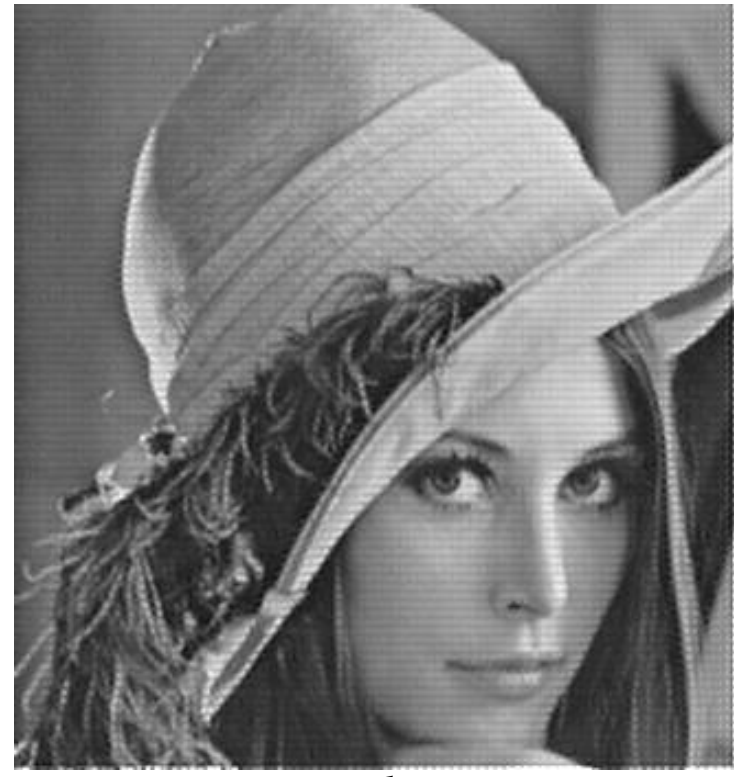

$d$

Fig. 4. SR reconstructed Lena images in case of sequence of frames corrupted by salt\&pepper noise. a) $L_{2}+\mathrm{BTV}$ technique. b) Lorentzian+BTV technique. c) Huber+Tikhonov technique. d) Lorentzian+Tikhonov technique. 
Table IV: Numerical results in case of noiseless frames for the two scenes.

\begin{tabular}{|l|c|c|}
\hline \multirow{2}{*}{\multicolumn{1}{c|}{ SR method }} & Xydeas and Petrovich/MSE \\
\cline { 2 - 3 } & Lena & Stanford \\
\hline$L_{2}+$ Tikhonov & $0.8927 / 0.0039$ & $0.8738 / 0.0090$ \\
\hline$L_{2}+\mathrm{BTV}$ & $0.9138 / 0.0036$ & $0.8759 / 0.0088$ \\
\hline$L_{1}+\mathrm{Tikhonov}$ & $0.8976 / 0.0028$ & $0.8739 / 0.0084$ \\
\hline$L_{1}+\mathrm{BTV}$ & $0.9139 / 0.0026$ & $0.8751 / 0.0082$ \\
\hline Huber+Tikhonov & $0.9044 / 0.0029$ & $0.8788 / 0.0087$ \\
\hline Huber+BTV & $0.9225 / 0.0026$ & $0.8825 / 0.0085$ \\
\hline Lorentzian+Tikhonov & $0.9056 / 0.0020$ & $0.8813 / 0.0082$ \\
\hline Lorentzian+BTV & $0.9234 / 0.0019$ & $0.8842 / 0.0080$ \\
\hline
\end{tabular}

Table V: Numerical results in case of frames corrupted by salt\&pepper noise for the two scenes.

\begin{tabular}{|l|c|c|}
\hline \multirow{2}{*}{\multicolumn{1}{c|}{ SR method }} & \multicolumn{2}{c|}{ Xydeas and Petrovich/MSE } \\
\cline { 2 - 3 } & Lena & Stanford \\
\hline$L_{2}+$ Tikhonov & $0.8007 / 0.0803$ & $0.7852 / 0.1478$ \\
\hline$L_{2}+\mathrm{BTV}$ & $0.8046 / 0.0734$ & $0.7864 / 0.1364$ \\
\hline$L_{1}+\mathrm{Tikhonov}$ & $0.8998 / 0.0028$ & $0.8705 / 0.0086$ \\
\hline$L_{1}+\mathrm{BTV}$ & $0.9126 / 0.0026$ & $0.8739 / 0.0084$ \\
\hline Huber+Tikhonov & $0.8889 / 0.0029$ & $0.8509 / 0.0109$ \\
\hline Huber+BTV & $0.8938 / 0.0028$ & $0.8518 / 0.0106$ \\
\hline Lorentzian+Tikhonov & $0.9044 / 0.0028$ & $0.8771 / 0.0087$ \\
\hline Lorentzian+BTV & $0.9240 / 0.0025$ & $0.8823 / 0.0085$ \\
\hline
\end{tabular}

Table VI: Numerical results in case of frames corrupted by Gaussian noise for the two scenes.

\begin{tabular}{|l|c|c|}
\hline \multirow{2}{*}{\multicolumn{1}{|c|}{ SR method }} & Xydeas and Petrovich/MSE \\
\cline { 2 - 3 } & Lena & Stanford \\
\hline$L_{2}+$ Tikhonov & $0.7898 / 0.0486$ & $0.7547 / 0.0139$ \\
\hline$L_{2}+\mathrm{BTV}$ & $0.8162 / 0.0157$ & $0.7600 / 0.0136$ \\
\hline$L_{1}+\mathrm{Tikhonov}$ & $0.8103 / 0.0187$ & $0.7602 / 0.0138$ \\
\hline$L_{1}+\mathrm{BTV}$ & $0.8241 / 0.0083$ & $0.7646 / 0.0136$ \\
\hline Huber+Tikhonov & $0.8337 / 0.0069$ & $0.7816 / 0.0140$ \\
\hline Huber+BTV & $0.8379 / 0.0032$ & $0.7828 / 0.0144$ \\
\hline Lorentzian+Tikhonov & $0.8294 / 0.0077$ & $0.7735 / 0.0136$ \\
\hline Lorentzian+BTV & $0.8344 / 0.0045$ & $0.7753 / 0.0138$ \\
\hline
\end{tabular}


Table VII: Numerical results in case of frames corrupted by speckle noise for the two scenes.

\begin{tabular}{|l|c|c|}
\hline \multirow{2}{*}{\multicolumn{1}{|c|}{ SR method }} & \multicolumn{2}{c|}{ Xydeas and Petrovich/MSE } \\
\cline { 2 - 3 } & Lena & Stanford \\
\hline$L_{2}+$ Tikhonov & $0.7852 / 0.0807$ & $0.7530 / 0.0146$ \\
\hline$L_{2}+\mathrm{BTV}$ & $0.8074 / 0.0279$ & $0.7547 / 0.0141$ \\
\hline$L_{1}+\mathrm{Tikhonov}$ & $0.8163 / 0.0127$ & $0.7571 / 0.0151$ \\
\hline$L_{1}+\mathrm{BTV}$ & $0.8254 / 0.0071$ & $0.7584 / 0.0149$ \\
\hline Huber+Tikhonov & $0.8235 / 0.0104$ & $0.7775 / 0.0153$ \\
\hline Huber+BTV & $0.8348 / 0.0046$ & $0.7783 / 0.0155$ \\
\hline Lorentzian+Tikhonov & $0.8214 / 0.0108$ & $0.7718 / 0.0155$ \\
\hline Lorentzian+BTV & $0.8338 / 0.0046$ & $0.7727 / 0.0156$ \\
\hline
\end{tabular}

Table VIII: Numerical results in case of frames corrupted by Poisson noise for the two scenes.

\begin{tabular}{|l|c|c|}
\hline \multirow{2}{*}{\multicolumn{1}{c|}{ SR method }} & \multicolumn{2}{c|}{ Xydeas and Petrovich/MSE } \\
\cline { 2 - 3 } & Lena & Stanford \\
\hline$L_{2}+$ Tikhonov & $0.8303 / 0.0139$ & $0.7565 / 0.0133$ \\
\hline$L_{2}+\mathrm{BTV}$ & $0.8361 / 0.0107$ & $0.7719 / 0.0131$ \\
\hline$L_{1}+\mathrm{Tikhonov}$ & $0.8317 / 0.0095$ & $0.7728 / 0.0126$ \\
\hline$L_{1}+\mathrm{BTV}$ & $0.8341 / 0.0086$ & $0.7836 / 0.0123$ \\
\hline Huber+Tikhonov & $0.8560 / 0.0023$ & $0.8006 / 0.0125$ \\
\hline Huber+BTV & $0.8567 / 0.0023$ & $0.8045 / 0.0126$ \\
\hline Lorentzian+Tikhonov & $0.8548 / 0.0034$ & $0.7886 / 0.0119$ \\
\hline Lorentzian+BTV & $0.8563 / 0.0033$ & $0.8004 / 0.0118$ \\
\hline
\end{tabular}

\subsection{Evaluation and discussion}

\subsubsection{Grouping per common data-fidelity term}

Table IX presents ranking, in descending order of performance, for the SR methods grouped per common data-fidelity term. The particular ranking is extracted from the numerical results given in the tables of subsection 5.1 and holds for noiseless frames as well as all types of noisy frames. Grouping the SR methods per common data-fidelity term it makes clear that in a pair of methods, which employ the same error norm for the similarity cost, the method with the BTV regularizer predominates over the method with the Tikhonov regularizer regardless of the presence or absence of noise in frames. The methods under comparison present common estimator in the data-fidelity term so the ranking assessment is determined by the priors utilized for the regularization term. The BTV regularization is a robust regularization technique capable of performing adaptive image smoothing. Thus, it produces sharp edges and preserves point like details. Unlike BTV case, the smoothing performed by the Tikhonov technique removes noisy and edge pixels both. So, the resulting image lacks 
sharp edges and point like details. Consequently, the BTV regularized SR method performs superiorly to the Tikhonov regularized method.

Table IX: Ranking in performance (descending order) for the SR methods grouped per common datafidelity term, in case of both noiseless and noisy frames.

\begin{tabular}{|l|c|}
\hline $\begin{array}{c}\text { Methods grouped per common } \\
\text { data-fidelity term/Data }\end{array}$ & $\begin{array}{c}\text { Noiseless } \\
\text { and noisy }\end{array}$ \\
\hline$L_{2}+$ Tikhonov & 2 \\
\hline$L_{2}+$ BTV & 1 \\
\hline$L_{1}+$ Tikhonov & 2 \\
\hline$L_{1}+$ BTV & 1 \\
\hline Huber+Tikhonov & 2 \\
\hline Huber+BTV & 1 \\
\hline Lorentzian+Tikhonov & 2 \\
\hline Lorentzian+BTV & 1 \\
\hline
\end{tabular}

\subsubsection{Grouping per common regularization term}

Table X presents performance ranking in descending order for the tested methods grouped per common regularization term, as mandated by the tables of subsection 5.1. for the various cases of data. After having grouped the super-resolution techniques per common regularization term, in general terms the conclusion that the ranking in performance is in accordance with the ranking in robustness of the estimators employed for the data-fidelity term can be reached. The compared techniques present common regularization term. Thus, the estimators used for the data-fidelity term determine performance ranking. Actually, the particular ranking can be explained in terms of the intrinsic differences between the utilized estimators. Specifically, the Lorentzian error norm gives outliers weights of decreasing values according to their magnitude. With regard to the Huber norm, it gives all outliers a constant weight of one. Therefore, the Huber norm treats all outliers equally. As far as the $L_{1}$ and $L_{2}$ estimators are concerned, they treat all measurements equally thus do not pay any special attention to outliers. In fact, the $L_{2}$-norm estimation is much more sensitive to outliers than the $L_{1}$ estimation as it gives them too much influence. Consequently, the Lorentzian and Huber estimators are first and second, respectively, in robustness ranking. The $L_{1}$ estimator per- 
forms inferiorly to the Huber estimator. The worst performance is exhibited by the $L_{2}$ error norm.

In particular, when the employed sequence of frames is noiseless the ranking in methods performance is in full accordance with the ranking in robustness of the estimators utilized for the data-fidelity term. Therefore, the Lorentzian and Huber methods are first and second, respectively, in performance. The SR techniques employing the $L_{1}$ estimator perform inferiorly to the Huber estimator techniques. The worst performance is exhibited by the methods which utilize the $L_{2}$ error norm for the similarity cost. Nevertheless, there are some differentiations in performance ranking as far as the cases of noisy frames are concerned. This can be attributed to the relation, which exists between the model of the distributional characteristic of the noise and the model of the estimator employed for assuring fidelity to the data, that affects the SR reconstruction performance.

Specifically, when the sequence of frames is corrupted by salt\&pepper noise, performance ranking differentiation from the noiseless case appears in the comparison of the Huber with the $L_{1}$ estimator. Actually, the Huber estimator performs inferiorly to the $L_{1}$ estimator in the presence of salt\&pepper noise in the LR frames sequence. This can be attributed to the quadratic form of the Huber error norm. Thus, the Lorentzian techniques exhibit the best performance. The techniques utilizing the $L_{1}$ estimator in the data-fidelity term come second in performance. Actually, the $L_{1}+\mathrm{BTV}$ and $L_{1}+$ Tikhonov methods predominate over the $\mathrm{Hu}-$ ber+BTV and Huber+Tikhonov methods, respectively. The techniques with the $L_{2}$ estimator exhibit the worst performance.

In case of frames corrupted by Gaussian, speckle or Poisson noise, differentiation in performance ranking from the noiseless frames case appears in the comparison of the Huber with the Lorentzian estimator. In fact, the Huber estimator performs superiorly to the Lorentzian estimator. The Huber error norm, due to its dual nature of $L_{2}$ and $L_{1}$ estimation, manages to treat the Gaussian, speckle and Poisson noise models more efficiently than the Lorentzian error norm. Therefore, the methods employing the Huber error norm in the data-fidelity term perform best. In fact, the Huber+BTV and Huber+Tikhonov methods exhibit better performance than the Lorentzian+BTV and Lorentzian+Tikhonov methods, respectively. The $L_{1}$ methods perform inferiorly to the Lorentzian methods, while the methods utilizing the $L_{2}$ estimator perform worst. 
Table X: Ranking in performance (descending order) for the SR methods grouped per common regularization term, in case of noiseless and noisy frames.

\begin{tabular}{|l|c|c|c|}
\hline $\begin{array}{c}\text { Methods grouped per common } \\
\text { regularization term/Data }\end{array}$ & Noiseless & Salt\&pepper & $\begin{array}{c}\text { Gaussian, speckle } \\
\text { or Poisson }\end{array}$ \\
\hline$L_{2}+$ BTV & 3 & 4 & 4 \\
\hline$L_{1}+$ BTV & 2 & 3 & 3 \\
\hline Huber+BTV & 1 & 1 & 2 \\
\hline Lorentzian+BTV & 4 & 4 & 4 \\
\hline$L_{2}+$ Tikhonov & 3 & 2 & 3 \\
\hline$L_{1}+$ Tikhonov & 2 & 3 & 1 \\
\hline Huber+Tikhonov & 1 & 1 & 2 \\
\hline Lorentzian+Tikhonov & & & 1 \\
\hline
\end{tabular}

\subsubsection{Grouping per pairs}

Conclusions are also reached by grouping the methods per pairs, each of which exhibits rival terms with different robustness of efficiency. Table XI regards the particular grouping of methods. Actually, this table presents the ranking in performance, as mandated by the numerical results of the tables of subsection 5.1, for the SR methods under comparison in each pair.

\section{a) Noiseless frames}

In case of employing noiseless sequence of frames the difference in robustness of efficiency, between the rival estimators employed for the data-fidelity term, plays a vital role in determining ranking in performance for the SR methods under comparison in each pair. More specifically, if the particular robustness difference is great, the best performance is exhibited by the method which utilizes the most robust estimator in the data-fidelity term. Otherwise, thus in case of small robustness difference between the estimators, the method with the most robust regularization term comes first in performance.

So, in pairs 1, 3, 5 and 6 the difference in robustness of efficiency between the rival estimators utilized for the similarity cost is not as great as to determine performance ranking. Particularly, in pairs 1 and 3 the Huber estimator rivals the $L_{2}$ and $L_{1}$ estimators, respectively. But, the Huber error norm presents the dual nature of $L_{2}$ and $L_{1}$ estimation. Regarding pairs 5 and 6, the Lorentzian and $L_{1}$ estimators compete with the Huber and $L_{2}$ estimators, respectively. However, the particular rival estimators are sequential in robustness ranking. Thereafter, in pairs $1,3,5$ and 6 the ranking assessment is determined by the priors utilized for the regulari- 
zation term. Actually, the best performance is exhibited by the method which employs the most robust regularization technique. Thus, the Huber+Tikhonov technique performs inferiorly to the $L_{2}+\mathrm{BTV}$ and $L_{1}+\mathrm{BTV}$ techniques. Additionally, the $L_{1}+$ Tikhonov and Lorentzian+Tikhonov techniques perform worst than the $L_{2}+\mathrm{BTV}$ and Huber+BTV techniques, respectively.

With regard to pair 2, there is a great difference in robustness of efficiency between the rival estimators utilized for the data-fidelity term. Specifically, the Lorentzian estimator, first in performance ranking per robustness, competes with the $L_{2}$ estimator, last in robust performance ranking. Therefore, in this pair the error norm employed for measuring the difference between the projected estimate of the HR image and each LR frame determines performance ranking. In fact, the method with the most robust error norm in the data-fidelity term comes first in performance. So, the Lorentzian+Tikhonov technique predominates over the $L_{2}+\mathrm{BTV}$ technique.

As far as pair 4 is concerned, there is a disagreement between the two scenes, Lena and Stanford, regarding the SR techniques ranking in performance. In the particular pair the $L_{1}+\mathrm{BTV}$ technique is compared with the Lorentzian+Tikhonov technique. Actually, the $L_{1}+\mathrm{BTV}$ method predominates over the Lorentzian+Tikhonov method in case of the Lena scene. The contrary is true in case of the Stanford scene.

\section{b) Noisy frames}

In case of employing noisy sequence of frames the general conclusion that the ranking in performance, for the super-resolution techniques under comparison in each pair, is determined by the estimator utilized for the data-fidelity term can be reached. In fact, in each pair the method employing the most robust estimator, for assuring fidelity to the corrupted by noise data, comes first in performance. This can be attributed to the strong presence of outliers in the data. However, differentiations from the general conclusion appear in some methods behavior under certain types of noise present in the LR frames.

More specifically, when the employed sequence of frames is corrupted by salt\&pepper noise, in pairs 1, 2, 3, 5 and 6 the best SR performance is exhibited by the method which utilizes the most robust error norm for assuring fidelity to the noisy data. Therefore, the $L_{2}+$ BTV method performs inferiorly to the Huber+Tikhonov and Lorentzian+Tikhonov methods. Additionally, the $L_{1}+\mathrm{BTV}$ and Lorentzian+Tikhonov methods predominate over the 
Huber+Tikhonov and Huber+BTV techniques, respectively. Also, the $L_{1}+$ Tikhonov technique performs better than the $L_{2}+\mathrm{BTV}$ technique. Nevertheless, differentiation appears in the case of pair 4. Despite the fact that the $L_{1}$ estimator is less robust than the Lorentzian estimator, the $L_{1}+\mathrm{BTV}$ method predominates over the Lorentzian+Tikhonov method.

As far as experimentation with Poisson noisy frames is concerned, all pairs follow the general rule regarding performance ranking. Thus, the $L_{2}+\mathrm{BTV}$ method performs inferiorly to the Huber+Tikhonov and Lorentzian+Tikhonov methods. Also, the $L_{1}+\mathrm{BTV}$ technique exhibits worst performance than the Huber+Tikhonov and Lorentzian+Tikhonov techniques. Additionally, the $L_{1}$ estimator combined with the Tikhonov prior outperforms the $L_{2}$ estimator combined with the BTV prior. Regarding pair 5, the Huber+BTV technique predominates over the Lorentzian+Tikhonov technique.

Regarding experimentation with frames corrupted by Gaussian noise, in pairs 1, 2, 3, 4 and 5 first in SR performance comes the technique which utilizes the most robust estimator for the data-fidelity term. However, in pair 6 the contrary is true. Thus, the $L_{2}+\mathrm{BTV}$ method outperforms the $L_{1}+$ Tikhonov method. The ranking in performance for the SR techniques under comparison in pair 6 can be explained in terms of the relation between the model of the distributional characteristic of the noise and the model of the estimator assuring fidelity to the corrupted by Gaussian noise data. Actually, the Gaussian noise model is closer to the $L_{2}$ estimator model than to the $L_{1}$ estimator model.

When employing speckle noisy sequence of LR frames, in pairs 1, 2, 5 and 6 the best performance is exhibited by the method employing the most robust estimator for the similarity cost. So, the $L_{2}+\mathrm{BTV}$ technique performs worst than the Huber+Tikhonov, Lorentzian+Tikhonov and $L_{1}+$ Tikhonov techniques. Additionally, the Huber+BTV method outperforms the Lorentzian+Tikhonov method. Nevertheless, no common conclusion is reached for the two employed scenes Lena and Stanford in case of the pairs 3 and 4. Explicitly, the $L_{1}+$ BTV technique predominates over the Huber+Tikhonov and Lorentzian+Tikhonov techniques, respectively, in case of the Lena scene. The contrary is true in case of the Stanford scene. 
Table XI: Ranking in performance for the SR methods grouped per pairs, regarding the various cases of data (Lena/Stanford).

\begin{tabular}{|c|l|c|c|c|c|c|}
\hline Pair number & \multicolumn{1}{|c|}{ SR methods } & Noiseless & Salt\&pepper & Gaussian & Speckle & Poisson \\
\hline \multirow{3}{*}{1} & $L_{2}+\mathrm{BTV}$ & 1 & 2 & 2 & 2 & 2 \\
\cline { 2 - 7 } & Huber+Tikhonov & 2 & 1 & 1 & 1 & 1 \\
\hline \multirow{2}{*}{2} & $L_{2}+\mathrm{BTV}$ & 2 & 2 & 2 & 2 & 2 \\
\cline { 2 - 7 } & Lorentzian+Tikhonov & 1 & 1 & 1 & 1 & 1 \\
\hline \multirow{3}{*}{3} & $L_{1}+\mathrm{BTV}$ & 1 & 1 & 2 & $1 / 2$ & 2 \\
\cline { 2 - 8 } & Huber+Tikhonov & 2 & 2 & 1 & $2 / 1$ & 1 \\
\hline \multirow{3}{*}{4} & $L_{1}+\mathrm{BTV}$ & $1 / 2$ & 1 & 2 & $1 / 2$ & 2 \\
\cline { 2 - 8 } & Lorentzian+Tikhonov & $2 / 1$ & 2 & 1 & $2 / 1$ & 1 \\
\hline \multirow{2}{*}{5} & Huber+BTV & 1 & 2 & 1 & 1 & 1 \\
\cline { 2 - 8 } & Lorentzian+Tikhonov & 2 & 1 & 2 & 2 & 2 \\
\hline \multirow{2}{*}{6} & $L_{2}+$ BTV & 1 & 2 & 1 & 2 & 2 \\
\cline { 2 - 7 } & $L_{1}+$ Tikhonov & 2 & 1 & 2 & 1 & 1 \\
\hline
\end{tabular}

\subsubsection{Scene consideration}

In the present work experimentation is carried out with two different scenes Lena and Stanford. The Stanford scene is quite more demanding than the Lena scene, as it exhibits more fine details. Common conclusions are reached for the two different scenes, regarding tradeoffs between the data-fidelity and regularization terms in the SR image reconstruction techniques. However, different conclusions are drawn for the employed scenes in certain cases of grouping the methods per pairs. Specifically, comparing the $L_{1}+\mathrm{BTV}$ technique with the Lorentzian+Tikhonov technique, for noiseless frames and for frames corrupted by speckle noise, different ranking results are obtained for the two scenes. Additionally, comparing the $L_{1}+\mathrm{BTV}$ with the Huber+Tikhonov technique for frames corrupted by speckle noise, again different performance ranking is obtained for the scenes utilized. In the aforementioned cases for the most demanding scene, Stanford, the best performance is exhibited by the method employing the most robust estimator in the data-fidelity term. For the least demanding scene, Lena, the SR method with the most robust regularizer performs best.

In order to provide some intuition as to what extent the pair grouping conclusions could change when considering other scenes, a statistical evaluation is presented in Table XII. Actually, the particular table presents the percentage of experimental data which leads to common conclusions for the two scenes regarding each pair of SR techniques. Thereafter, in pairs 
1, 2, 5 and 6, 100\% of experimental data leads to common ranking for the methods performance. However, in pair 3 , where the $L_{1}+\mathrm{BTV}$ technique is compared with the $\mathrm{Hu}$ ber+Tikhonov technique, common performance ranking for the two scenes is assessed by $80 \%$ of the experimental data. As far as pair 4 is concerned, $L_{1}+\mathrm{BTV}$ technique versus Lorentzian+Tikhonov technique, common conclusions for the two different scenes are reached by only $60 \%$ of the data. In the particular pair of methods, $40 \%$ of the experimental data leads to different conclusions for the employed scenes.

Table XII: Percentage of experimental data which leads to common performance ranking conclusions for the two different scenes, regarding the grouping of methods per pairs.

\begin{tabular}{|c|c|c|}
\hline Pair number & SR methods & Data percentage \\
\hline \multirow{2}{*}{1} & $L_{2}+\mathrm{BTV}$ & \multirow{2}{*}{$100 \%$} \\
\hline & Huber+Tikhonov & \\
\hline \multirow{2}{*}{2} & $L_{2}+\mathrm{BTV}$ & \multirow{2}{*}{$100 \%$} \\
\hline & Lorentzian+Tikhonov & \\
\hline \multirow{2}{*}{3} & $L_{1}+\mathrm{BTV}$ & \multirow{2}{*}{$80 \%$} \\
\hline & Huber+Tikhonov & \\
\hline \multirow{2}{*}{4} & $L_{1}+\mathrm{BTV}$ & \multirow{2}{*}{$60 \%$} \\
\hline & Lorentzian+Tikhonov & \\
\hline \multirow[b]{2}{*}{5} & Huber+BTV & \multirow{2}{*}{$100 \%$} \\
\hline & Lorentzian+Tikhonov & \\
\hline \multirow{2}{*}{6} & $L_{2}+\mathrm{BTV}$ & \multirow{2}{*}{$100 \%$} \\
\hline & $L_{1}+$ Tikhonov & \\
\hline
\end{tabular}

\section{Conclusions}

In this work trade-offs between the data-fidelity and regularization terms, which formulate the SR image reconstruction problem in the context of stochastic regularized techniques, are discussed. Experimentation is carried out with the $L_{2}, L_{1}$, Huber and Lorentzian estimators employed for the data-fidelity term. In addition, the Tikhonov and BTV regularizers are utilized for the regularization term. Eight different SR techniques are formulated. Superresolution image reconstruction is performed employing noiseless frames as well as frames corrupted by noise of various models. The experimental results are obtained by grouping the SR methods into three groups. Comparisons in SR performance per the aforementioned 
groupings take place. The conclusions reached help to select an effective SR image reconstruction method, among several potential ones, for a given LR sequence of frames.

Grouping the methods per common data-fidelity term it makes clear that the best performance is exhibited by the method which employs the most robust regularization technique. The particular conclusion concerns noiseless frames as well as frames corrupted by noise of various models. When the methods are grouped per common regularization, in general terms it is concluded that the ranking in performance is in accordance with the ranking in robustness of the estimators employed for the data-fidelity term. Nevertheless, there are some differentiations from the general conclusion in the cases of noisy frames. Thereafter, in case of frames corrupted by salt\&pepper noise the $L_{1}$ estimator performs superiorly to the Huber estimator. Concerning those frames which are corrupted by Gaussian, speckle or Poisson noise, the Huber estimator predominates over the Lorentzian estimator. Conclusions can also be reached with regard to the grouping of methods per pairs. When the employed sequence of frames is noiseless the difference in robustness of efficiency, between the rival estimators utilized for the data-fidelity term, affects the ranking in performance for the techniques under comparison in each pair. Particularly, if this robustness difference is great, first in SR performance comes the method with the most robust estimator in the data-fidelity term. However, in case of small robustness difference between the estimators, the best performance is displayed by the technique with the most robust regularization. In case of sequence of frames corrupted by noise, in general terms it is concluded that the performance ranking, for the SR methods under comparison in each pair, is determined by the error norm employed for the similarity cost. Actually, in each pair first in SR performance comes the method with the most robust estimator in the similarity cost. Without any grouping considerations, the Lorentzian+BTV technique exhibits the best performance for noiseless frames and for frames corrupted by salt\&pepper noise. Nevertheless, when frames are corrupted by Gaussian, speckle or Poisson noise, the Huber+BTV technique outperforms all the other techniques.

\section{References}

[1] R. Y. Tsai, T. S. Huang, Multiframe image restoration and registration, ACVIP 1 (1984) 317-339.

[2] A. Panagiotopoulou, V. Anastassopoulos, Super-resolution image reconstruction employing Kriging interpolation technique, IWSSIP 2007 \& EC-SIPMCS 2007 Maribor Slovenia (2007) 151-154. 
[3] A. Panagiotopoulou, V. Anastassopoulos, Super-resolution reconstruction of thermal infrared images, $4^{\text {th }}$ WSEAS Int. Conf. on Remote Sensing Venice Italy (2008) 40-44.

[4] A. K. Katsaggelos, R. Molina, J. Mateos, Super Resolution of Images and Video, Synthesis Lectures on Image, Video and Multimedia Processing, Morgan and Claypool Publishers, USA, 2007.

[5] V. Tsagaris, A. Panagiotopoulou, V. Anastassopoulos, Interpolation in multispectral data using neural networks, SPIE Conf. 5573 (2004) 460-470.

[6] A. Panagiotopoulou, V. Anastassopoulos, Scanned images resolution improvement using neural networks, Neural Comput. Appl. 17 (2008) 39-47.

[7] S. C. Park, M. K. Park, M. G. Kang, Super-resolution image reconstruction: A technical overview, IEEE SP Magazine 20 (2003) 21-36.

[8] S. Farsiu, M. D. Robinson, M. Elad, P. Milanfar, Fast and robust multiframe superresolution, IEEE Trans. on Image Processing 13 (2004) 1327-1344.

[9] L. C. Pickup, S. J. Roberts, A. Zisserman, A sampled texture prior for image superresolution, Advances in NIPS Conf. (2004).

[10] V. Patanavijit, S. Jitapunkul, A robust iterative multiframe super-resolution reconstruction using a Huber-Bayesian approach with Huber-Tikhonov regularization, ISPACS (2006) 13-16.

[11] V. Patanavijit, S. Jitapunkul, An iterative super-resolution reconstruction of image sequences using a Bayesian approach and affine block-based registration, EUSIPCO (2006).

[12] V. Patanavijit, S. Jitapunkul, An iterative super-resolution reconstruction of image sequences using affine block-based registration, IWCMC (2006) 51-56.

[13] V. Patanavijit, S. Jitapunkul, A Lorentzian stochastic estimation for a robust iterative multiframe super-resolution reconstruction with Lorentzian-Tikhonov regularization, EURASIP JASP (2007) 1-21 .

[14] A. Panagiotopoulou, V. Anastassopoulos, Regularized super-resolution image reconstruction employing robust error norms, OE 48 (2009) 117004-1 to 117004-14.

[15] S. Farsiu, D. Robinson, M. Elad, P. Milanfar, Advances and challenges in superresolution, Int. J. Imag. Syst. Technol. 14 (2004) 47-57.

[16] J. Tukey, Understanding Robust and Exploratory Data Analysis, John Wiley and Sons, USA, 1983.

[17] M. J. Black et al., Robust anisotropic diffusion, IEEE Trans. on Image Processing 7 (1998) 421-432. 
[18] H. W. Engl, M. Hanke, A. Neubauer, Regularization of Inverse Problems, Kluwer Academic Publishers, Netherlands, 2000.

[19] A. Neumaier, Solving ill-conditioned and singular linear systems: A tutorial on regularization, SIAM Review 40 (1998) 636-666.

[20] N. Nguyen, P. Milanfar, G. Golub, Efficient generalized cross-validation with applications to parametric image restoration and resolution enhancement, IEEE Trans. on Image Processing 10 (2001) 1299-1308.

[21] N. K. Bose, S. Lertrattanapanich, J. Koo, Advances in superresolution using L-curve, ISCAS 20012 (2001) 433-436.

[22] M. Belge, M E Kilmer, E. L Miller, Efficient determination of multiple regularization parameters in a generalized L-curve framework, Inverse Problems 18 (2002) 1161-1183.

[23] M. V. W. Zibetti, F. S. V. Bazan, J. Mayer, Determining the regularization parameters for super-resolution problems, Signal Processing 88 (2008) 2890-2901.

[24] T. Chan, S. Osher, J. Shen, The digital TV filter and nonlinear denoising, IEEE Trans. on Image Processing 10 (2001) 231-241.

[25] M. Elad, On the origin of the bilateral filter and ways to improve it, IEEE Trans. on Image Processing 11 (2002) 1141-1151.

[26] C. S. Xydeas, V. Petrovic, Objective image fusion performance measure, Electron. Lett. 36 (2000) 308-309. 